\title{
Genetic Programming
}

National Cancer Institute

\section{Source}

National Cancer Institute. Genetic Programming. NCI Thesaurus. Code C19418.

A method for obtaining an optimal programming algorithm. Thousands of randomly created programs are progressively evolved over time using an evolutionary search based on the principle of survival of the fittest. Mechanisms include the equivalent of recombination, mutation, duplication, deletion, etc. 